\title{
An Infant with Congenital Heart Block and SARS-CoV-2 Infection: Challenges in Diagnosis and Management
}

Suresh K Angurana ${ }^{1} \odot$, Ashish Agarwal ${ }^{2}$, Indranil Biswas ${ }^{3}$, Atit A Gawalkar ${ }^{4}$, Vivek Jaswal ${ }^{5}$, Vishnu Koneru ${ }^{6}$, Kavitha TK ${ }^{7}$, Manoj K Rohit ${ }^{8}$, Goverdhan D Puri ${ }^{9} \odot$, Muralidharan Jayashree ${ }^{10} \odot$

\begin{abstract}
The coronavirus disease-2019 (COVID-19) pandemic posed a major challenge for delivery of health care services especially for non-COVID-19 related diseases. We present a 1-year-old girl who was symptomatic for last 3 months with recurrent myoclonic jerks, the cause for which could not be evaluated due to lockdown and disruption of healthcare services. She presented to us with complete heart block, cardiogenic shock, and acute kidney injury. The management included mechanical ventilation, placement of an internal jugular venous and peritoneal catheter, insertion of transvenous pacing lead, and later on an epicardial permanent pacemaker implantation.

Keywords: Congenital heart block, COVID-19, Invasive procedures, Personal protective equipment.

Journal of Postgraduate Medicine, Education and Research (2022): 10.5005/jp-journals-10028-1557
\end{abstract}

\section{INTRODUCTION}

Congenital heart block $(\mathrm{CHB})$ is a rare disorder with an incidence of around 1/15,000-20,000 live births. ' It usually occurs secondary to passive transplacental transfer of anti-Ro and anti-La antibodies which damage the conduction system of the heart. Anti-Ro antibodies are most prevalent in mothers of affected infants (85\%) followed by anti-La antibodies (55\%) and $>50 \%$ of the mothers are usually asymptomatic. ${ }^{2}$ If diagnosis and treatment is delayed in neonatal period, $\mathrm{CHB}$ is associated with high morbidity and mortality with $5-30 \%$ cases develop dilated cardiomyopathy without pacing.

The nationwide lockdown to cut the transmission of SARS-CoV-2 transmission has created many hurdles in the delivery of healthcare services especially for non-COVID-19 illnesses ${ }^{4}$ as highlighted by this case. Also, we highlighted the challenges faced while performing various invasive procedures and interventions while donning the personal protective equipment (PPEs).

\section{Case Description}

A 1-year-girl with smooth perinatal period was noted to have bradycardia in the neonatal period which was not evaluated further. At 9th month, she started with brief myoclonic jerks for which phenytoin was started from a peripheral health center without further evaluation with no clinical response. In first week of July 2020, she developed high grade fever, respiratory distress, loose stools, and increased frequency of myoclonic jerks. She was taken to a private hospital where she was found to have respiratory failure, hypotensive shock, encephalopathy, and complete heart block on electrocardiogram. She was started on mechanical ventilation, vasoactive support, and referred to our center for further management.

At presentation, the heart rate was $55 /$ minute with idioventricular rhythm on ECG (Fig.1A), well palpable pulses, capillary refill time 3 seconds, blood pressure $78 / 50 \mathrm{~mm} \mathrm{Hg}$, and $\mathrm{SpO}_{2} 98 \%$ on positive pressure ventilation $\left(40 \% \mathrm{FiO}_{2}\right)$. She was started on isoprenaline infusion, and planned for pacemaker insertion. The child tested positive, and mother and father negative for SARS-CoV-2 RT-PCR. She was shifted to the dedicated Pediatric
$1,2,6,7,10$ Department of Pediatrics, Postgraduate Institute of Medical Education and Research, Chandigarh, India

3,9 Department of Anaesthesiology and Intensive Care, Postgraduate Institute of Medical Education and Research, Chandigarh, India

${ }^{4,8}$ Department of Cardiology, Advanced Cardiac Centre, Postgraduate Institute of Medical Education and Research, Chandigarh, India

${ }^{5}$ Department of Cardiovascular and Thoracic Surgery, Postgraduate Institute of Medical Education and Research, Chandigarh, India

Corresponding Author: Muralidharan Jayashree, Department of Pediatrics, Postgraduate Institute of Medical Education and Research, Chandigarh, India, Phone:+91 7087008311,e-mail:mjshree@hotmail.com

How to cite this article: Angurana SK, Agarwal A, Biswas I, et al. An Infant with Congenital Heart Block and SARS-CoV-2 Infection: Challenges in Diagnosis and Management. J Postgrad Med Edu Res 2022;56(1):34-36.

Source of support: Nil

Conflict of interest: None

COVID-19ICU. The echocardiography showed large patent ductus arteriosus (PDA) with left-to-right shunt and ejection fraction of $25-30 \%$. In view of hemodynamic improvement, the temporary pacemaker insertion was deferred and planned for permanent epicardial pacemaker implantation.

But over next 24 hours, there was gradual fall in heart rate, onset of hypotensive shock, oliguria, and high anion gap metabolic acidosis. Left internal jugular venous access was secured for vasoactive support (epinephrine and milrinone), peritoneal dialysis was established, and emergency transvenous pacemaker was inserted through right internal jugular venous route with full PPEs (Fig. 1C).

Following transvenous pacing, heart rate (Fig. 1B), hemodynamics, renal functions, and acidosis improved; she was weaned from vasoactive support, peritoneal dialysis, and mechanical ventilation; and extubated on day 16 . Later, she underwent single chamber (VVIR) epicardial permanent pacemaker implantation. The single pacing lead was fixed on the epicardial surface of right ventricle through subxiphoid approach and battery generator

() The Author(s). 2022 Open Access This article is distributed under the terms of the Creative Commons Attribution 4.0 International License (https://creativecommons. org/licenses/by-nc/4.0/), which permits unrestricted use, distribution, and non-commercial reproduction in any medium, provided you give appropriate credit to the original author(s) and the source, provide a link to the Creative Commons license, and indicate if changes were made. The Creative Commons Public Domain Dedication waiver (http://creativecommons.org/publicdomain/zero/1.0/) applies to the data made available in this article, unless otherwise stated. 


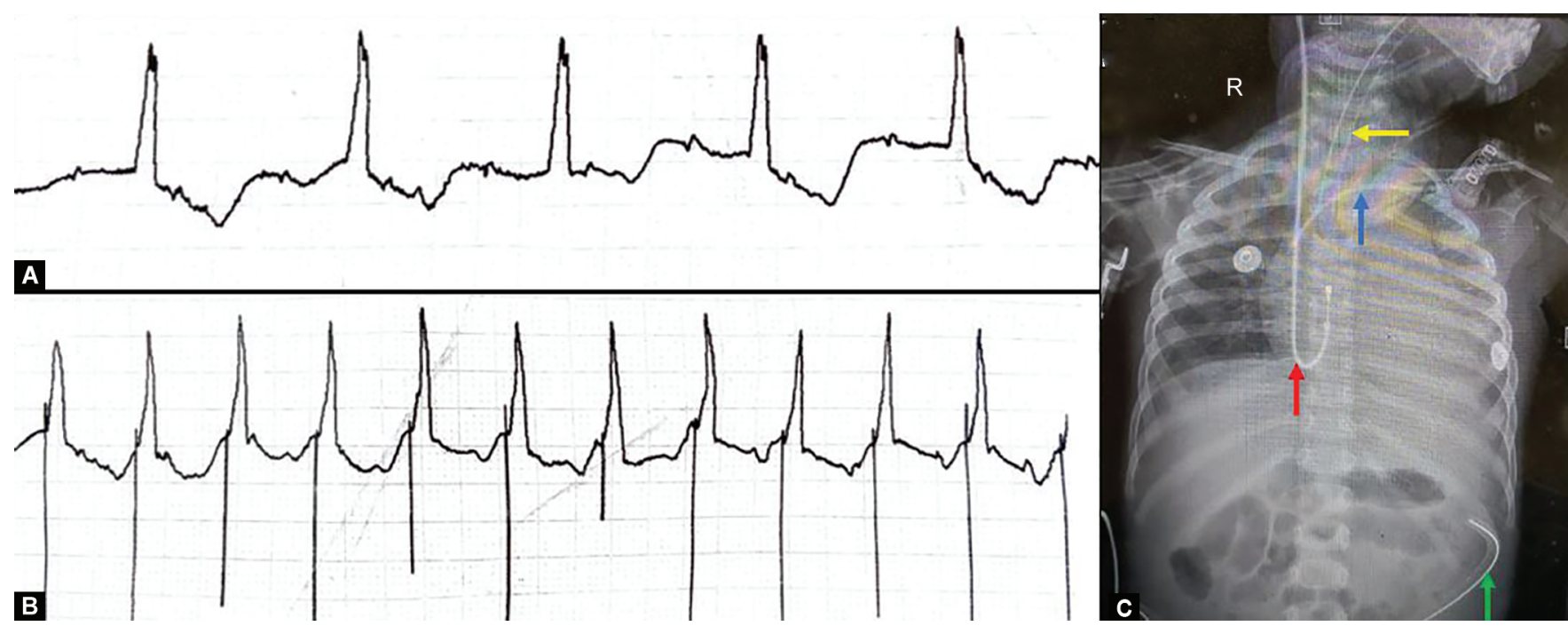

Figs 1 A to C: (A) ECG showing bradycardia with idioventricular rhythm; (B) ECG after transvenous pacing; (C) Chest radiograph showing in situ endotracheal tube (yellow arrow), right subclavian line (blue arrow), transvenous pacing lead with sheath (red arrow), and peritoneal dialysis catheter (green arrow)

was placed in the abdominal wall below rectus abdominus muscle over the peritoneum. PDA ligation and transfixation was done simultaneously through left posterolateral thoracotomy via 4th intercostal space. Following the procedure, she had normal hemodynamics. Her mother tested positive for anti-Ro antibodies ( $>240 \mathrm{IU} / \mathrm{mL}$ ) and negative for anti-La antibodies. At 6-month follow-up, she was gaining developmental milestones, with no seizure recurrence, and had normal hemodynamic parameters.

\section{Discussion}

Due to COVID-19 pandemic, the healthcare services for noncommunicable diseases have been severely disrupted due to lockdown, limited mobility, lack of transport facilities, limited access to healthcare facilities, cancellation of all elective care, and major shift in focus to COVID-19 illness. ${ }^{4}$ To mitigate the disruption of healthcare services, the WHO recommends the strengthening of telemedicine and triage services. Telemedicine services are critical for delivery of nonurgent health services where direct contact between patient and health care provider is not required. ${ }^{5} \mathrm{~A}$ well-designed triage system will ensure that right care is provided to the right patients at the right time. ${ }^{6}$

Thus, the opportunity to identify myoclonic jerks due to $\mathrm{CHB}$ in index child was missed at the first contact and diagnosis was delayed. Underlying CHB, brief duration of myoclonic jerks, and poor response to antiepileptics suggest that these were possibly cardiac in origin and manifestation of syncope. The use of phenytoin (acts on ion channels) could have aggravated the bradycardia due to the negative chronotropic and dromotropic action on sick heart. ${ }^{3}$ Although we had not performed electroencephalography, the semiology was that of myoclonic seizures.

She presented with complete heart bock, cardiogenic shock, and acute kidney injury. The management challenges included performing various invasive procedures (central venous cannulation, peritoneal dialysis, and transvenous pacing) while wearing PPEs (gloves, coverall/jump suits, goggles, face shield, N-95 mask, shoe covers, and head cover) as recommended by the WHO and the Ministry of Health and Family Welfare while caring for COVID-19 patient admitted to an ICU. ${ }^{7,8}$ However, it is difficult to perform invasive procedures while donned in full PPE.
An international online survey involving 30 countries highlighted the various problems encountered while working with full PPE, use of double or triple gloved hands affected performance of manual tasks, face shields and goggles generated glare, goggles and face shield become foggy hampering the visibility, and respirator and face shield disrupted voice impairing communication. ${ }^{9}$ There is possibility of increase in adverse events, breach in safety protocols, and asepsis bundles while performing invasive procedures with PPEs. We faced similar challenges while performing invasive interventions in this index child.

The conduction system abnormalities in a neonate or infant born to mother with anti-Ro and anti-La antibodies ranges from prolongation of PR interval to complete heart block, with development of progressive cardiomyopathy in severe cases. The cardiac system abnormalities can be detected in-utero by 16th week of gestation. It usually occurs secondary to passive transplacental transfer of anti-Ro and anti-La antibodies which damage the conduction system of the heart. However, their presence alone is not sufficient to cause the disease as only $2 \%$ offspring born to mothers with anti-Ro and anti-La antibodies develop complete heart block.

Because maternal autoantibodies cross placenta and gain access to fetus by 16th week of gestation, all pregnant women with anti-Ro and anti-La antibodies or with history of offspring with complete heart block or neonatal lupus should be monitored by regular fetal electrocardiography from 16th week onwards. If fetal bradycardia is detected unexpectedly during gestation, maternal anti-Ro and anti-La antibodies should be done. A mother who has born a child with CHB caused by neonatal lupus has $17 \%$ (approximately) risk of recurrence with subsequent pregnancies. Several drugs (dexamethasone, betamethasone, intravenous immunoglobulin, plasmapheresis, hydroxychloroquine, and terbutaline) has been tried in pregnant women with anti-Ro and anti-La antibodies to prevent the occurrence or progression of fetal cardiac abnormalities including $\mathrm{CHB}$, hydrops fetalis, and endocardial fibroelastosis.

Significant conduction system abnormalities after birth should be treated with cardiac pacing and occasionally with intravenous immunoglobulin and steroids. Infants with conduction system abnormalities without cardiomyopathy had excellent outcome 
with cardiac pacing. If conduction system disturbances are not addressed, there infants may progress to exercise intolerance, cardiomyopathy (which may require cardiac transplantation), arrhythmias, and death.

\section{Conclusion}

The disruption of healthcare services has huge impact on the care of non-COVID-19 illnesses which can be mitigated by strengthening telemedicine and triage services. Performing various invasive procedures with PPE is a challenge which can be mitigated by coordinated efforts of a multidisciplinary team, timely planning of the procedure, use of checklists, and communication.

\section{ORCID}

Suresh K Angurana • https://orcid.org/0000-0001-6370-8258 Goverdhan D Puri ๑ https://orcid.org/0000-0002-9763-4055 Muralidharan Jayashree ๑ https://orcid.org/0000-0002-6149-1355

\section{References}

1. Baruteau AE, Pass RH, Thambo JB, et al. Congenital and childhood atrioventricular blocks: pathophysiology and contemporary management. Eur J Pediatr 2016;175(9):1235-1248. DOI: 10.1007/ s00431-016-2748-0

2. Johnson B. Overview of neonatal lupus. J Pediatr Health Care 2014;28(4):331-341. DOI: 10.1016/j.pedhc.2013.07.015
3. Bergfeldt L. Differential diagnosis of cardiogenic syncope and seizure disorders. Heart 2003;89(3):353-358. DOI: 10.1136/ heart.89.3.353

4. World Health Organization Preliminary results: rapid assessment of service delivery for noncommunicable diseases during the COVID-19 pandemic. 29 May 2020. https://www.who.int/who-documentsdetail/rapid-assessment-of-service- delivery-for-ncds-duringthecovid-19-pandemic

5. Chauhan V, Galwankar S, Arquilla B, et al. Novel coronavirus (COVID-19): leveraging telemedicine to optimize care while minimizing exposures and viral transmission. J Emerg Trauma Shock 2020;13(1):20-24. DOI:10.4103/JETS.JETS_32_20

6. Maves RC, Downar J, Dichter JR, et al. Triage of scarce critical care resources in COVID-19 an implementation guide for regional allocation: an expert panel report of the task force for mass critical care and the American College of Chest Physicians. Chest 2020;158(1):212-225. DOI: 10.1016/j.chest.2020.03.063

7. World Health Organization Rational use of personal protective equipment for Coronavirus Disease 2019 (COVID-19) and considerations during severe shortages. 2020. https://apps.who. int/iris/bitstream/handle/10,665/331,215/WHO-2019-nCov-IPCPPE use2020.1-eng.pdf

8. Ministry of Health and Family Welfare, Directorate General of Health Sciences. Novel Coronavirus Disease 2019 (COVID-19): Guidelines on rational use of Personal Protective Equipment. https://www.mohfw.gov. in/pdf/GuidelinesonrationaluseofPersonalProtectiveEquipment.pdf

9. Benítez CY, Güemes A, Aranda J, et al. Impact of personal protective equipment on surgical performance during the COVID-19 pandemic. World J Surg 2020;44(9):2842-2847. DOI: 10.1007/s00268020-05648-2 